\title{
Pratiques endogènes de gestion de l'eau au service des politiques d'adaptation aux changements climatiques dans la région du lac Tchad
}

\author{
Armel Sambo \\ Faculté des Arts, Lettres et Sciences Humaines, Université de Maroua, BP 644, Cameroun \\ Correspondence: Armel Sambo (samboarmel@yahoo.fr) \\ Published: 16 November 2021
}

\begin{abstract}
Résumé. Dans un contexte-marqué par la variabilité climatique comme la région du lac Tchad, il est utile de prendre en compte et de revaloriser les stratégies d'adaptation aux phénomènes induits par les Changements Climatiques (CC), dans le but d'améliorer la gestion durable des ressources en eau. Cette étude vise donc à identifier et à interpréter les pratiques locales de gestion et d'accès à l'eau, des dynamiques de celles-ci, et son importance dans la cadre de mises en œuvre des projets d'adaptation aux changements climatiques dans la région du lac Tchad. La méthodologie adoptée a consisté d'abord à la collecte des données (par des entretiens, observation, etc.) auprès des populations riveraines du lac Tchad, ensuite à leur traitement analyse et enfin la rédaction de cet article. Ainsi, quelques techniques issues de savoir faire traditionnel et local, ont été identifiées et sont susceptibles d'être revalorisées dans un cadre stratégique. Il s'agit : l'existence des mares d'eau sacrée, la gestion collective des mares d'eau naturelles, la gestion communautaire des pêcheries, l'irrigation, etc. Toutes ces pratiques et techniques ont été inventées et ou améliorées par les populations pour répondre à des contraintes de production qu'ils connaissent bien.
\end{abstract}

\section{Introduction}

Le changement climatique risque d'amplifier les vulnérabilités socio-économiques auxquelles les populations font face, et particulièrement dans la zone du lac Tchad où l'économie est essentiellement basée sur l'agriculture, l'élevage et la pêche. C'est pourquoi, les travaux de terrain doivent focaliser l'attention non seulement sur les effets du changement climatique dans le bassin du lac Tchad mais aussi sur les pratiques d'adaptation aux variations environnementales qui peuvent être développées dans le cadre de la mise en place des projets de développement à base communautaire. Ainsi, l'objectif principal de ce travail est de savoir quelles sont les pratiques endogènes que les populations ont développé pour assurer une gestion durable des ressources en eau dans la zone du lac Tchad et comment peut- on les mettre en pratique à travers des projets communautaires dans le cadre des plans nationaux d'adaptation au Changement climatique en Afrique? Ce retour d'expérience souligne l'intérêt - la nécessité - d'identifier, répertorier/recenser/classer ces pra- tiques, et d'en mesurer/d'évaluer l'impact/la valeur ajoutée sur la gestion durable et rationnelle des ressources en eau.

La méthode adoptée a consisté d'abord à la collecte des données (par des entretiens, observation, etc.) auprès des populations riveraines du lac Tchad, ensuite à leur traitement analyse et enfin la rédaction de cette communication. D'abord, elle présente le niveau et la vulnérabilité des populations aux effets du Changement Climatique, ensuite les stratégies endogènes de gestion rationnelle des eaux qu'elles ont développées et enfin, l'intérêt de l'intégration des ses savoirs locaux dans le processus du développement durable de la région du lac Tchad.

\section{La région et les populations du bassin du lac Tchad affectées par le Changement Climatique}

Les rapports du GIEC indiquent que la région du lac Tchad compte parmi les zones les plus affectées par le changement climatique (GIEC, 2007). L'on peut observer ce phénomène à 
travers la baisse de la pluviosité, l'assèchement du lac Tchad et la raréfaction des ressources en eau (Sambo, 2013).

Les populations riveraines sont de plus en plus vulnérables à ces modifications survenant dans leur environnement. L'évolution de la pluviométrie de ces 50 dernières années démontrent à suffisance de la baisse de la pluviosité. Elle est marquée par la chute la plus importante et la plus durable de sa pluviométrie. Une comparaison des isohyètes des années 1950, «considérées comme la décennie la plus humide, la plus sèche étant celles des années 1990, a montré un déplacement considérable vers le sud »(Olivry et al., 1996). La baisse de la pluviométrie a atteint près de $100 \mathrm{~mm}$ pour tous les $100 \mathrm{~km}$ de distance en dépit des variations annuelles et spatiales (CBLT, 2000).

Les variations de la pluviométrie se sont répercutées sur les écoulements les affluents du lac Tchad (Logone, Chari, etc.) et sur la quantité d'eau du lac. Le lac Tchad est donc en constante régression vu son évolution historique. Entre le XII $e$ et le XVIII $e$ siècles, le lac Tchad va connaître une baisse catastrophique des ressources en eau qui va conduire à son assèchement successivement au XVe et à la fin du XVIII $e$ siècle (Fadimatou Dia, 2006, pp. 15-16). Depuis le XIXe et le XXe siècles, le bassin du lac Tchad a connu plusieurs épisodes de sécheresse. Le lac Tchad avait en 1963 une superficie d'environ $25000 \mathrm{~km}^{2}$ et suite à la sécheresse de 1973 , sa superficie en juillet n'était que de $9000 \mathrm{~km}^{2}$ (Beauvilain, 1989, p. 92). Depuis 2013, l'on observe une crue abondante en saison de pluies. Les images ci- dessous produites par la NASA sont contestées par certains chercheurs. Ils pensent que le lac Tchad, loin de s'inquiéter sur sa disparition est juste passé «à l'état de petit Tchad, comme il l'a été plusieurs fois au cours des 150 dernières années ». Depuis 2013, l'on constate que le lac a atteint une superficie inondée de $14800 \mathrm{~km}^{2}$ (Lemoalle et al., 2015). Toutefois, cette variabilité est influencée aussi ces dernières années par le changement climatique.

La dégradation du niveau de vie des populations, victimes des effets du changement climatique est une réalité dans la zone du lac Tchad. Toutefois les changements climatiques ne sont pas à la base de toutes les difficultés rencontrées dans cette zone écologique. En effet des facteurs anthropiques, comme la construction des barrages, la déforestation, ou les problèmes politiques liés à un encadrement efficace du monde rural, sont aussi responsables de la pauvreté des populations. Plus de $80 \%$ des personnes interrogées établissent une relation entre ces transformations environnementales et la baisse des ressources en eau, et partant de la production agro - sylvo - pastorale ce d'autant plus que l'économie dans cette localité est essentiellement rurale (Sambo, 2013).

\section{Les populations ont développé des stratégies de gestion rationnelle}

Dans la majorité des cas, l'adaptation aux changements climatiques s'inscrit naturellement dans un cadre de développement puisque tous les pays s'efforcent d'une manière ou d'une autre de gérer les mêmes systèmes sociaux, écologiques et économiques en vue d'instaurer un développement durable (Mohamadou Sall et al., 2011). Il s'agit de répertorier, de classifier et des prioriser les stratégies d'adaptation qu'on peut soutenir financièrement. De ce fait donc, l'adaptation intègre les politiques environnementales des Etats africains. Il est important de noter que les communautés locales ont toujours adopté des stratégies et mesures afin de s'adapter aux conséquences des modifications des ressources en eau liées à celles du climat. Il s'agit entre autres selon ce tableau ci-dessous :

La prise en compte de ces pratiques dans un cadre stratégique comme on le constate dans ce tableau sera un atout énorme pour améliorer la résilience des populations du bassin du lac Tchad au changement climatique. C'est ainsi qu'il importe pour les Etats d'intégrer ces savoirs faire par la mise en place des projets de développement. Les pratiques les plus courantes observées dans la région et qui sont au centre des activités des ONG sont : la construction des mares d'eau, des infrastructures de rétention d'eau, l'organisation de la transhumance, l'irrigation, etc.

De plus en plus, l'on observe un changement dans les pratiques qui se modernisent. Ainsi, aux pratiques traditionnelles qui existent beaucoup plus en zones rurales à savoir entre autres l'aménagement des puits à ciel ouvert, l'usage des cours d'eau à des fins de lessive et bain, etc. se développent les aménagements modernes tels que les forages et les puits modernes. Ainsi, ces Pratiques et connaissances endogènes peuvent servir de mécanismes de gestion rationnelle et durables des ressources en eau, en partant des stratégies d'adaptation aux changements climatiques dans cette partie de l'Afrique.

\section{La prise en compte de certaines des pratiques endogènes dans la politique environnementale est une nécessité}

Les pratiques endogènes dans le domaine de gestion des ressources en eau sont donc des précieuses ressources qui peuvent contribuer à améliorer la base du processus de décision locale dans des domaines tels que la sécurité alimentaire, la gestion des ressources et par d'autres activités économiques. Sur cette base, il est possible de co-construire et de concevoir avec les populations du bassin du lac Tchad des systèmes techniques améliorés issus de les propres savoirfaire, afin de gérer rationnellement et durablement les ressources en eau sans bouleverser le monde rural.

Dans le processus de gestion des ressources en eau, la construction des mares d'eau, des puits, le dévelop- 

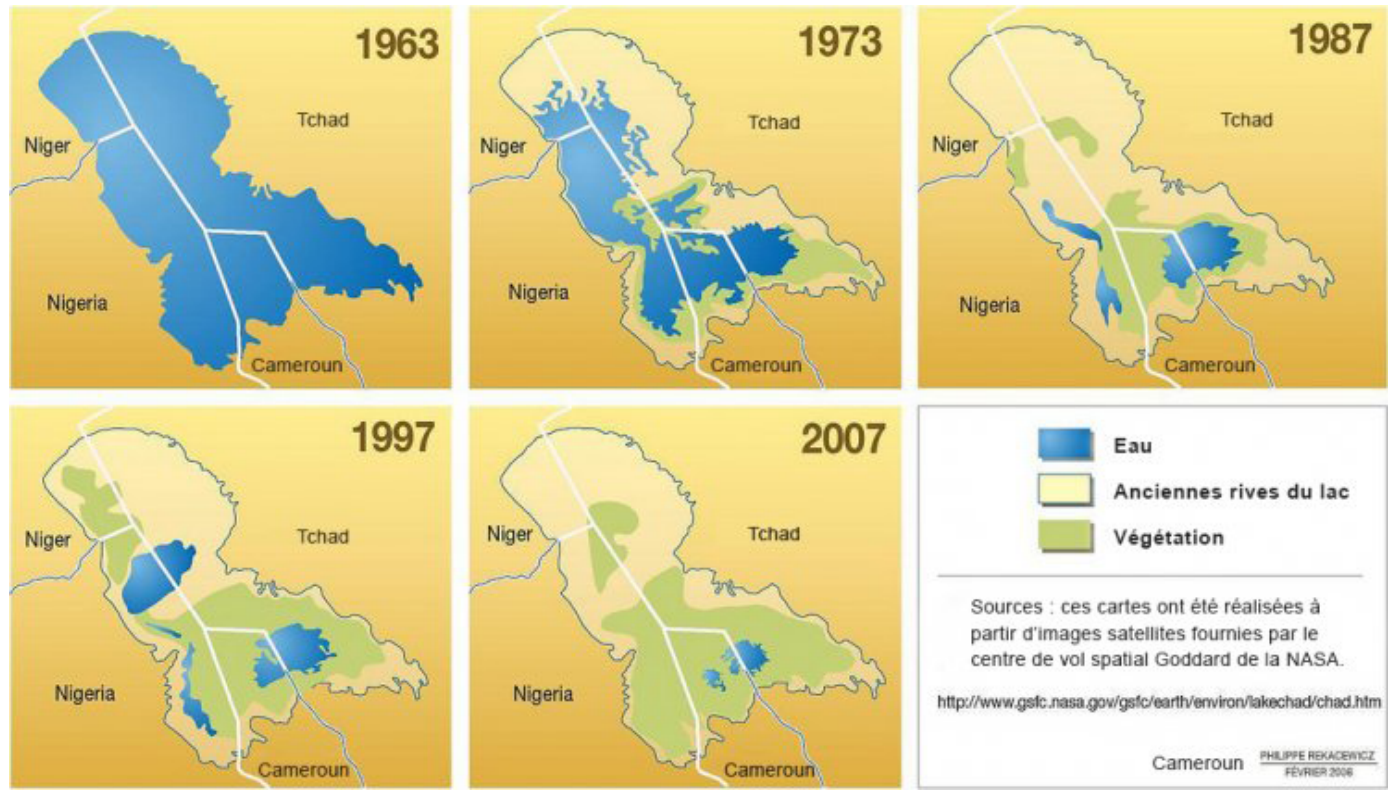

Figure 1. Evolution de l'étendue en eau du lac Tchad de 1967 à 2007 (source : Documentation de la CBLT, 2010).

Tableau 1. Les stratégies d'adaptation de gestion rationnelle des ressources en eau (Source : Enquête de terrain, 2012).

\begin{tabular}{|c|c|c|c|}
\hline Systèmes & Impact du CC & $\begin{array}{l}\text { Pratiques endogènes d'adaptation au } \\
\text { CC }\end{array}$ & $\begin{array}{l}\text { Les cadres stratégiques dans lesquels } \\
\text { intégrer ces savoirs }\end{array}$ \\
\hline \multirow[t]{3}{*}{ Agriculture } & $\begin{array}{l}\text { Variabilité } \\
\text { pluviométrique }\end{array}$ & $\begin{array}{l}\text { réaménagement des } \\
\text { calendriers agricoles }\end{array}$ & $\begin{array}{l}\text { Mise à la disposition des paysans des don- } \\
\text { nées sur la pluviométrie. } \\
\text { Sensibilisation sur l'évolution des calen- } \\
\text { driers de la pluviométrie }\end{array}$ \\
\hline & $\begin{array}{l}\text { Diminution des } \\
\text { ressources en eau }\end{array}$ & L'irrigation pour la culture & $\begin{array}{l}\text { Gestion rationnelle des mares d'eau, } \\
\text { aménagements des ouvrages } \\
\text { hydroagricoles, etc. }\end{array}$ \\
\hline & & $\begin{array}{l}\text { Recharge artificielle des nappes } \\
\text { souterraines }\end{array}$ & $\begin{array}{l}\text { Développement des infrastructures de } \\
\text { rétention d'eau pour la recharge des eaux } \\
\text { souterraines }\end{array}$ \\
\hline \multirow[t]{3}{*}{ Élevage } & Raréfaction de l'eau & transhumance & Organiser la transhumance \\
\hline & & Préservation des mares d'eau & $\begin{array}{l}\text { Construction des mares d'eau artificielles } \\
\text { (rétention des eaux de pluie). } \\
\text { Renforcement des capacités des structures } \\
\text { de gestion des mares }\end{array}$ \\
\hline & & Gestion des mares d'eau naturelles & Construction des mares d'eau artificielles \\
\hline \multirow[t]{2}{*}{ Pêche } & $\begin{array}{l}\text { Baisse de stock } \\
\text { de poissons }\end{array}$ & gestion communautaire des pêcheries, & $\begin{array}{l}\text { Appui financier des populations à travers } \\
\text { des associations } \\
\text { Renforcer les capacités des communautés }\end{array}$ \\
\hline & & la préservation des mares d'eau sacrée & Revaloriser de cette tradition \\
\hline
\end{tabular}


pement des systèmes d'irrigation dans la vallée du Logone, etc. contribuent à augmenter la production agro-sylvopastorales (Sambo, 2018). Quelques techniques issues de savoir faire traditionnel et local, ont été identifiées et sont susceptibles d'être revalorisées dans un cadre stratégique. Il s'agit : l'existence des mares d'eau sacrée (stratégie de préservation des ressources), la gestion collective des mares d'eau naturelles, la gestion communautaire des pêcheries, des systèmes de recharge artificielle des nappes souterraines, etc. Quelques exemples (gestion rationnelle des mares d'eau, développement des infrastructures de rétention d'eau pour la recharge des eaux souterraines, construction des mares d'eau artificielles, etc.) mis en œuvre par les Organisations paysannes et certaines associations locales ont prouvé leur importance dans le renforcement de résilience des populations riveraines du lac Tchad.

Il existe plusieurs programmes et projets en cours financés par des organismes bilatéraux et multilatéraux pour aider les pays africains à gérer les risques liés aux changements climatiques ainsi qu'à s'adapter à la variabilité climatique. Seuls quelques-uns de ces programmes et projets traitent des risques liés à la gestion des ressources en eau partagées par plusieurs Etats. Pourtant, la gestion rationnelle des ressources naturelles doit faire partie intégrante des ripostes aux changements climatiques.

\section{Conclusions}

En conclusion, ces résultats nous permettent de dire que les populations riveraines du lac Tchad ont développées des mécanismes endogènes de gestion de l'eau qui peuvent - ou pourraient - jouer un rôle primordial dans la gouvernance environnementale. C'est une contribution qui met à profit les «propres savoir faire» des communautés locales dans le choix des politiques environnementales des Etats africains. Ce faisant, les décideurs ont en main un outil important de prise de décision qui peut améliorer la gestion intégrée des ressources eau du lac Tchad, en partant l'adaptation au changement climatique pour le développement des activités économiques liés à l'eau. Il est important dans ce contexte d'encourager la mise en place des projets communautaires et favoriser des conditions permettant aux pratiques communautaires et locales innovantes d'être élevées à des niveaux de mise en œuvre régionaux et nationaux.
Intérêts concurrents. The contact author has declared that there are no competing interests.

Clause de non-responsabilité. Publisher's note : Copernicus Publications remains neutral with regard to jurisdictional claims in published maps and institutional affiliations.

Déclaration du numéro spécial. This article is part of the special issue "Hydrology of Large River Basins of Africa". It is a result of the 4th International Conference on the "Hydrology of the Great Rivers of Africa", Cotonou, Benin, 13-20 November 2021.

\section{Références}

Beauvilain, A. : Nord Cameroun : crises et peuplement, T.1 et T.2, thèse de Doctorat es Lettres et Sciences humaines, Université de Rouen, 1989.

CBLT : Gestion intégrée du bassin fluvial, les défis du bassin du lac Tchad, vision 2025, Audit préparé par Steveland Consult, Norway, 2000.

Fadimatou Dia : La Commission du Bassin du Lac Tchad : 19642004, Mémoire de Maîtrise d'Histoire, Université de Ngaoundéré, 2006.

GIEC : Climate change 2007 : The physical science basis, Cambridge University Press, Cambridge, 2007.

Lemoalle, J. and Magrin, G. (Dir.) : Le développement du lac Tchad : situation actuelle et futurs possibles, Marseille, IRD Editions, coll. Expertise collégiale, bilingue français-anglais, 2014.

Mohamadou Sall, Serigne Mansour Tall, Aly Tandian, et M Al Assane Samb : Changements climatiques : stratégies d'adaptation et mobilités. Evidence à partir de quatre sites au Sénégal, GIZ, accessible à l'adresse suivante : http://pubs.iied.org/10612IIED. html, consulté le 11 février 2011.

Olivry, J.-C. : Hydrologie du lac Tchad, ORSTOM, Paris, 1996.

Sambo, A. : Perceptions locales et pratiques d'adaptation au changement climatique dans la gestion rationnelle des ressources en eau du Lac Tchad, in : Geo-Eco-Trop (Revue internationale de géologie, de géographie et d'écologie tropicales), No. 37, Tome, 2, 293-302, 2013.

Sambo, A. : Les savoirs endogènes comme stratégies d'adaptation au changement climatique chez les agriculteurs, les éleveurs et les agropasteurs de l'Extrême Nord du Cameroun, in : Revue Science et Technique, Lettres, Sciences Humaines et Sociales, Spécial hors-série No. 4, Ouagadougou, Burkina Faso, 11-25, 2018. 